\title{
Hybrid Power Supply System Monitoring And Control By Using Least Mean Square Algorithm
}

\author{
Prakash Thapa, Gye Choon Park, Sung Gi Kwon, Jin Lee and Na Kil Ju
}

\begin{abstract}
Due to the influence of different parameters, the electricity generated by the proton exchange membrane (PEM) fuel cell is not continuous. So, to provide the dynamic response for variable load and to meet the transient as well as peak load demand PEM fuel cell is connected with Li-ion Battery and made a hybrid power supply system. Such type of hybrid system has zero environmental effects and also solve the fossil fuel energy crisis in every country. But in this type of hybrid system, temperature effects is one of the most significant parameters which directly influence the performance of the system. So, in this paper, we are trying to investigate the detail design and effect of temperature parameters on hybrid system. Also, we are trying to monitoring, analyze and control the temperature effects through monitoring and control unit. The real time data communication for remote monitoring and control of hybrid system, a LMS estimation is used. By the use of this method, probability of energy signal detection will be increased as a result the frequency shortage problem will be solved. So the monitoring system hanging out and slow speed problem will be solved. Also from the control unit, temperature effect is controlled as per the system requirement. As a result, hybrid system generates maximum electricity with better performance.
\end{abstract}

Keywords-Combine PTH Sensor, CRN Network, Li-ion Battery, LMS estimation, Monitoring System, PEM fuel cell, Temperature Effect,

\section{Introduction}

In recent years, due to the decreasing levels of fossil fuel and increasing concerns about environmental pollution and global warming, the importance of renewable energy is growing rapidly. In this paper, we used hybrid PEM fuel cell and Li-ion battery system as a hybrid renewable energy. Hybrid power systems are used to supply loads and ensure load demand without interruption. This hybrid system consists of 50 watt PEM fuel cell stack, Li-ion battery and bidirectional converter and dc and ac load.

Prakash Thapa (Author)

line 1: Mokpo National University

line 2: South Korea

Gye Choon Park (Correspondant Author)

line 1: Mokpo National University

line 2: South Korea

Sung Gi Kwon (Co. Author)

line 1: Mokpo National University

line 2: South Korea

Jin Lee (Co. Author)

line 1: Mokpo National University

line 2: South Korea

Na Kil Ju (Co. Author)

line 1: Mokpo Science University

line 2: South Korea
PEM fuel cell has high power density so it can supports variable loads but due to the temperature, pressure, flow rate and humidity effect, the output of PEM fuel cell is not stable. So, a standalone PEM fuel cell system may not be satisfying the transient state and peak load demand condition. Thus, PEM fuel cell is combined with li-ion battery to provide the steady state power to variable load conditions. The proposed hybrid system can create maximum power with fast dynamic response and meet the transient as well as peak load demand.

In this paper, performance of hybrid system is measured theoretically and practically with variable temperatures. Also, in monitoring system, performance is measured by increased number of users. By the use of LMS estimation in cognitive environments, secondary frequency spectrum is used and solved the frequency shortage problem. So the maximum number of user can be used same monitoring system one at a time.

\section{PEM Fuel Cell Model}

A fuel cell is an electrochemical energy conversion device that directly converts hydrogen and oxygen into electrical energy and generates heat and water as a byproducts. PEM fuel cell are lighter, smaller and more efficient than other types of fuel cell. The demand of fuel cell is increased rapidly due to the high power density, low operating temperature, and fast start-up characteristics [1]. Fuel cell are being commercialized to provide electricity to buildings like hospital, school, houses to replace batteries in portable electronic devices, and as replacements for internal combustion engines in vehicles. The schematic diagram of PEM fuel cell is shown in [figure 1].

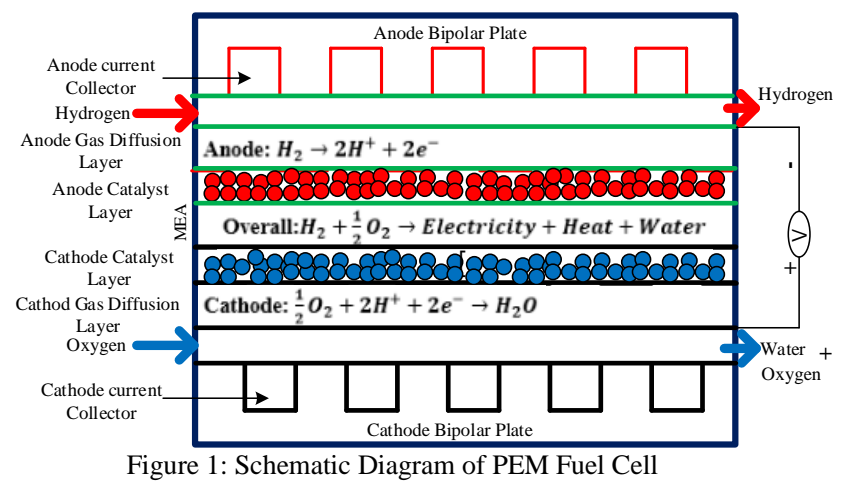

PEM fuel cell is also called proton exchange membrane fuel cells (PEMFC) which uses a thin $(50 \mathrm{~mm})$ proton conductive polymer membrane as the electrolyte. The catalyst is typically platinum supported on carbon with loadings of about $0.3 \mathrm{mg} / \mathrm{cm} 2$. In PEM fuel cell hydrogen side is positive and is called anode while the oxygen is passes through the negative terminal and is called cathode. Hydrogen fuel is processed at the anode where electrons are separated from protons on the surface of a platinum based 
catalyst. The protons pass through the membrane to the cathode side of the cell while the electrons travel in an external circuit by generating the electrical output of the cell. On the cathode side, another precious metal electrode combines the protons and electrons with oxygen to produce water, which is expelled as waste product.

The electrochemical reactions in PEM fuel cell occurs simultaneously on both anode and cathode sides of the membrane. The basic reactions occur in PEM fuel cell are [2]:

$$
\begin{aligned}
& \text { At anode: } \mathrm{H}_{2} \rightarrow 2 \mathrm{H}^{+}+2 e^{-} \\
& \text {At Cathode: } \frac{1}{2} \mathrm{O}_{2}+2 \mathrm{H}^{+}+2 e^{-} \rightarrow \mathrm{H}_{2} \mathrm{O} \\
& \text { Overall Reaction: } \mathrm{H}_{2}+\frac{1}{2} \mathrm{O}_{2} \rightarrow \mathrm{H}_{2} \mathrm{O}+\text { heat. }
\end{aligned}
$$

The output power of the PEM fuel cell is a product of stack voltage and current which is given by

$$
P_{f c}=V_{\text {Stack }} I
$$

The nominal voltage of each PEM fuel cell is 0.5 to 1.2 $\mathrm{V}$ at nominal power. So, total stack potential is a sum of the stack voltages or the product of the average cell potential and number of cells in the stack, Which is given by the following equation;

$$
\begin{gathered}
V_{\text {Stack }}=n \times E_{\text {Cell }} \\
E_{\text {Cell }}=E_{\text {rev }}-V_{\text {act }}-V_{\text {ohm }}-V_{\text {Con }}
\end{gathered}
$$

Where,

$$
\begin{aligned}
E_{\text {rev }}= & 1.229-0.85 \times 10^{-3}(T-298.15)+4.31 \times \\
& 10^{-5} T\left[\ln \left(P_{H 2}\right)+\frac{1}{2} \ln \left(P_{O 2}\right)\right]
\end{aligned}
$$

Where $\mathrm{P}$ and $\mathrm{T}$ represent the effective pressure and temperature respectively. Due to the irreversible losses in thermodynamic potential $\left(E_{r e v}\right)$, the actual potential $\left(E_{C e l l}\right)$ is decreased.

\section{Li-Ion Batter with Equivalent Circuit Model}

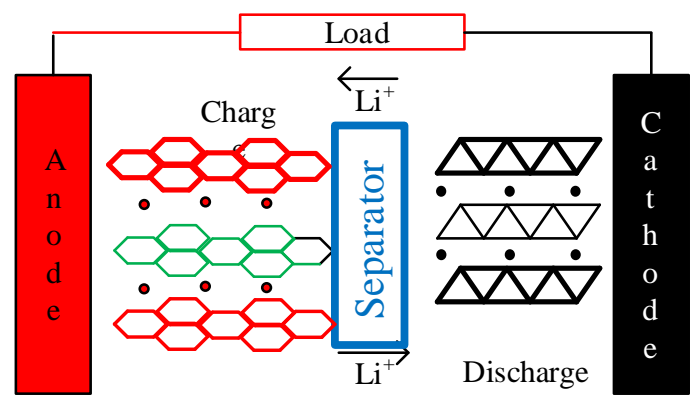

Figure 2: Schematic Diagram of Li-ion Battery

Li-ion battery is an electrochemical device which is used to stored electrical energy and used as per our requirement. [Figure 2] shows the simple block diagram of Li-ion cell which contains three main parts: a negative plate, positive plate and separator [3]. All three components are immersed in an electrolyte of a lithium salt such as LiPF6, LiBF4, or LiClO4.Generally, graphite is used as negative plate which is connected to the negative terminal whereas positive plate is connected to the positive terminal of the cell and is made from a mixture of several metal oxides such as LixMn2O4 and LixCoO2 [4]. The separator is a solid or liquid solution with a high concentration of lithium ions. It is an electrical insulator that prevents electrons from flowing between negative and positive plates, but the electrolyte allows ions to pass through it.

The equivalent RC model of li-ion battery is shown is [Figure 3]. Voltage source $V_{O C}$ stands for open circuit voltage, $V_{\text {out }}$ is the terminal output voltage and R0 is internal ohmic resistance of battery.

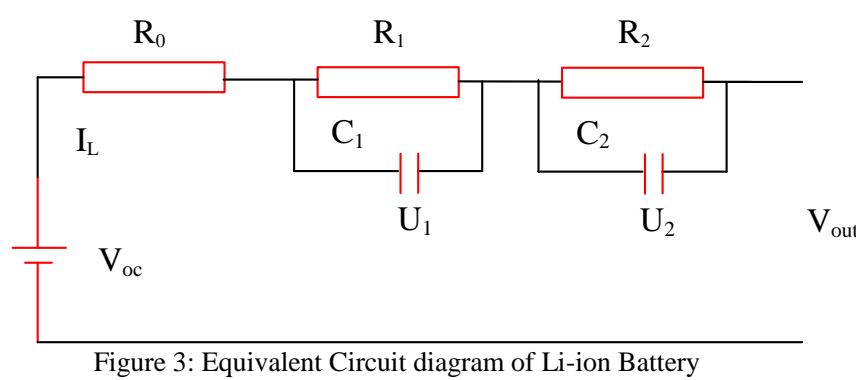

The open circuit voltage can be expressed in terms of state of Charge (SOC), which is given by the following equation [5];

$$
S O C=V_{O C}=F(z)=\sum_{i=0}^{n} C_{i} Z^{i}
$$

Where, $\mathrm{Z}=$ State of Charge, $\mathrm{n}=$ number or order of polynomial curve, $C_{i}$ = coefficient of polynomial coefficient

The mathematical form of second order RC model can be expressed by the following equation;

$$
\begin{aligned}
& V_{O C}=-\frac{I_{L}}{Q_{n}}+w_{1} \\
& \dot{U}_{1}=-\frac{U_{1}}{R_{1} C_{1}}+\frac{I_{L}}{C_{1}}+w_{2} \\
& \dot{U}_{2}=-\frac{U_{2}}{R_{2} C_{2}}+\frac{I_{L}}{C_{2}}+w_{3}
\end{aligned}
$$

By Solving, we get;

$$
V_{\text {out }}=V_{O C}-I_{L} R_{1}-U_{1}-U_{2}-R_{0} I_{L}+w
$$

Where, $Q_{n}=$ Capacity of battery, $I_{L}=$ load current, $V_{\text {out }}=$ output voltage of battery, $U_{1}, U_{2}=$ capacitive voltage, $V_{O C}=$ open circuit voltage, $\mathrm{w}=$ total noise.

Let's consider that, state vector $\mathrm{x}=\left(S O C, U_{1}, U_{2}\right)^{T}$, current $I_{L}$ and voltage $V_{\text {out }}$ are input, output variables then second order RC model for battery is given by the following equations;

$$
\begin{aligned}
x_{k+1} & =f\left(x_{k}, u_{k}\right)+w_{k-1} \\
y_{k} & =h\left(x_{k}, u_{k}\right)+v_{k}
\end{aligned}
$$

Where, $x_{k+1}$ is state vector at step time $\mathrm{k}, u_{k}=I_{L, k}$ is input vector, $y_{k}=V_{\text {out }, k}$ is observed output, $v_{k}=$ $\left(v_{1, k} v_{2, k} v_{3, k}\right)^{T}$ is process noise and $w_{k-1}$ is measured noise.

\section{Iv. Working Principle of Hybrid Energy System}

The hydrogen gas from cylinder goes to the fuel cell stack where hydrogen combine with ambient air and produce heat, water and electricity. The generated electricity is very lower capacity so we need to connect large number of stack with higher stack capacity.

In this model, each component is connected with monitoring and control unit through combine PTH sensor node and base station. We can observed monitor and control the temperature effect from monitoring and control unit. 
The communication block diagram of hybrid PEM fuel cell with li-ion battery system is shown in [figure 4].

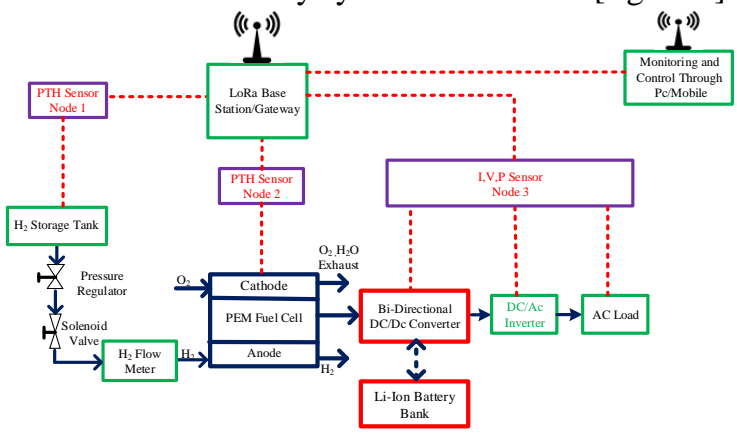

Figure 4: Communication Block Diagram of Hybrid PEM Fuel Cell and Liion Battery System

\section{v. LoRa Base Station}

LoRa technology is wide area, long range wireless technology that operates on industrial, scientific and medical (ISM) band frequency. There are different frequency bands defined in US (902 to $928 \mathrm{MHz}$ ), and South Korea (917 to $923 \mathrm{MHz}$ ) is used in LoRa wireless technology based network. It is physical layer protocol that offers a long range and low power communication medium for machine to machine (M2M) and IoT applications.

LoRa network consists of gateways, network servers and end devices. End devices are also known as notes and gateways are known as base stations or concentrators in LoRa network system. End devices and Gateways are connected wirelessly using ISM bands specified with single hob.

\section{vi. Combine PTH Digital Sensor (MS8607)}

The MS8607 is a combine digital sensor that can measures the temperature, pressure and humidity one at a time. The main feature of this device are low power consumptions, high accuracy and compactness. It has higher operating range of 10 to $2000 \mathrm{mbar}$ pressure, and 0 to $100 \% \mathrm{RH}$ humidity and $-40^{\circ} \mathrm{C}$ to $85^{\circ} \mathrm{C}$ temperature range.

Hydrogen cylinder is connected to LoRa base station through PTH Sensor Node 1. It can measure the pressure, temperature and humidity of hydrogen from storage tank and transmit that value to the monitoring unit through base station. Likewise, PTH Sensor Node 2 is connected between PEM fuel cell stack and LoRa base station. It can measure the pressure, temperature and humidity of fuel cell stack and transmit that value to the monitoring unit through base station. In the monitoring and control unit we observe that measured value and try to control PTH value to increase the performance of the fuel cell system. For the better performance fuel cell temperature should be $40^{\circ} \mathrm{C}$ to $85^{\circ} \mathrm{C}$ and pressure should be below 3atm. And humidity should be higher than $90 \%$. Similarly, PIV sensor measures the voltage, current and power of the system and transmit to the monitoring unit through base station.

\section{COGNITIVE RADIO NETWORK (CRN) CONCEPT}

Maximum number of radio spectrum is allocated through licensing. So the existing spectrum is being scar day by day due to the increased number of users and wireless applications. The frequency of $2.4 \mathrm{GHz}$ and $5 \mathrm{GHz}$ are unlicensed spectrum which frequencies are used by public easily to operate wireless devices. This band is also called ISM band.

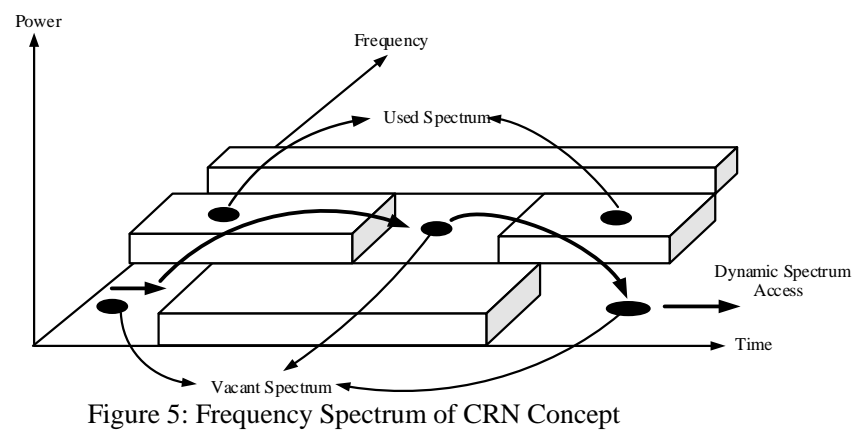

The vacant spectrum is also called white space which is used by cognitive radio temporarily. If this band is use by licensed user the cognitive radio moves to another vacant spectrum or stays in the same band, altering its transmission power level or modulation scheme to avoid interference [6].

Due to increase of radio environment frequency is divide into many areas. Some portion of that frequency is unused. Cognitive concept utilize that unlicensed frequency for enduser and provide them an autonomous system. Cogitative radio (CR) concept utilizes a large geographic area where end-user can be moveable.

The main features of CR has sensing ability of the surrounding radio environment and adjusting radio parameter where parameters include frequency, power, bandwidth, code rate, frame size, and encryption type. The objecting function [7] manipulate, control and change the parameters.

Let $P_{D}$ be the probability of detecting truly presenting signal on the considered frequency. A large desired detection of probability can be formulated as [8]

$$
P_{D}=P_{r}\left(M>\gamma_{E} \mid H\right)
$$

where $H: y(n)=u(n)+w(n) \quad$ Where $\mathrm{u}(\mathrm{n})$ is input signal and $M$ is waveform-based sensing metric [9]. The value of $\mathrm{M}$ can be obtained as

$$
M=\operatorname{Re}\left[\sum_{n=1}^{N} y(n) u^{*}(n)\right]
$$

\section{vIII. Least Mean Square (LMS) Estimation}

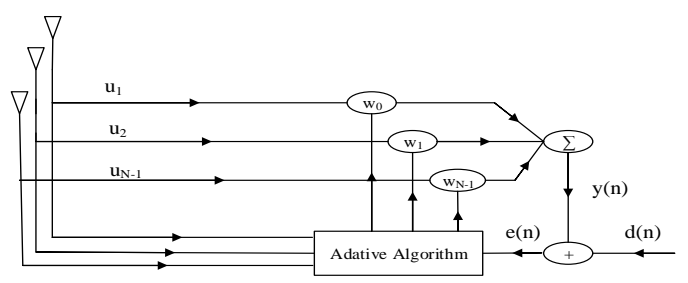

Figure 6: Block Diagram for RLS Estimation 
The LMS estimation is one of the important adaptive beam forming estimation for smart communication system which provides fastest convergence rate [10]-[11].

The linear combination of input vector $\mathrm{u}(\mathrm{n})$ and weight vector $w(n)$ is the output of uniform linear antenna $y(n)$ at any time $\mathrm{n}$ is given by

$$
y(n)=w^{H}(n) u(n)
$$

Where $w^{H}=\left[w_{1}, w_{2}, \ldots \ldots \ldots \ldots \ldots w_{N}\right]^{T}$

is the weight of matrix and

$u(t)=\left[u_{1}(n), u_{2}(n) \ldots \ldots \ldots \ldots\left[u_{N}(n)\right]^{T} \quad\right.$ is the Singular Matrix Vector. The error signal among sensor outputs can be calculated as ;

$$
\begin{aligned}
& e(n)=d(n)-y(n) \\
& y(n)=d(n)-e(n)
\end{aligned}
$$

From the method of Steepest descent, the weight vector $\mathrm{w}(\mathrm{n}+1)$ can be written as ;

$$
w(n+1)=w(n)+\frac{1}{2} \mu\left[-\nabla \mathrm{E}\left\{e^{2}(\mathrm{n})\right\}\right] . .
$$

Where, $\mu$ is the step size parameter, which controls the convergence characteristics of the LMS algorithm. Its value lies between 0 and 1 . And $e^{2}(\mathrm{n})$ is the mean square error (MSE) between the beam former output $y(n)$ and the reference which is given by;

$$
e^{2}(\mathrm{n})=\left[d^{*}(n)-w^{h} u(n)\right]^{2} .
$$

The gradient vector in the above updated weight equation can be computed as;

$$
\nabla\left(E\left\{e^{2}(n)\right\}\right)=-2 r+2 R w(n) \ldots
$$

In the steepest descent, the biggest problem is the computation involved in finding the values cross-correlation vector $r$ and auto correlation matrix $\mathrm{R}$ in real time. The LMS algorithm on the other hand simplifies this by using the instantaneous values of covariance matrices $r$ and $R$ instead of their actual values i.e.

$$
\begin{aligned}
& R(n)=u(n) u^{h}(n) \\
& r(n)=d^{*}(n) u(n)
\end{aligned}
$$

Therefore the updated weight can be calculated by the following equation;

$$
\begin{array}{r}
w(n+1)=\mathrm{w}(n)+\mu u(n)\left[d^{*}(n)-u^{h}(n) w(n) \ldots\right. \\
\mu=\frac{w(n+1)-\mathrm{w}(n)}{\mathrm{u}(n) e^{*}(n)} \ldots \ldots \ldots \ldots \ldots \ldots \ldots \ldots \ldots \ldots
\end{array}
$$

Where, $e^{*}(\mathrm{n})=\mu\left[d^{*}(n) u(n)-u(n) u^{H}(n)\right]$

The response of LMS algorithm is determined by three principal factors step size parameter, number of weights and eigenvalue of the correlation matrix of input data vector.

\section{LMS Estimation for Hybrid PEM Fuel Cell and Li-ION Battery System IN CRN Environment}

Smart antenna improves the performance of monitoring system and provides the better communication. It maximize antenna gain by the combination of diversity gain, array gain, and interface suppression by reducing multipath and interference which increase the data rate of user and maximum reception of desired direction signal while rejecting same frequency signal.

When smart LMS estimation is used, gateways are turning into the active user and provide autonomous environments. Let us consider, $L_{1}, L_{2} \ldots \ldots L_{S}$ be the number of load system which connected by wireless system to the Smart fuel cell and li-ion battery monitoring Network. Each gateways are connected to the number of sensor load system such as, $L_{11}, L_{12} \ldots . . L_{1 k}$. Let us consider we have equal number of sensor load appliances in each system. A sensor load matrix which has $L \times G$ in dimension. If a primary user $U_{N}$ is want to control one of his appliances $L_{11}$, end-user send a command which is encrypted and decrypted by smart energy network. AMI system is inbuilt and gets information about different load system that also share storage.

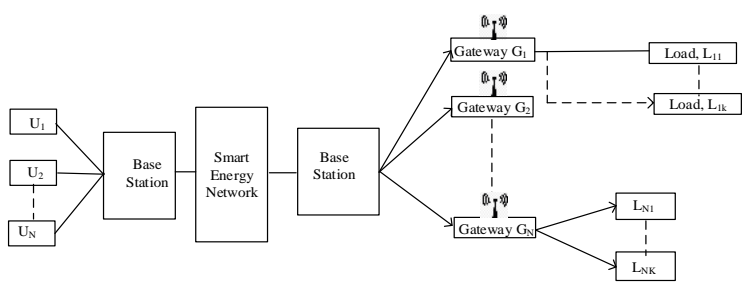

Figure 7: Smart Hybrid System Monitoring Network

Let we have $L_{1}, L_{2} \ldots . L_{S}$ be the number of load system which connected from wireless system to Smart energy Network (SEN). Each load system has different number load appliances such as $L_{11}, L_{12} \ldots \ldots L_{1 k}$. Let us consider we have equal number of load appliances in each system. A load matrix which has $L \times G$ in dimension. A primary user $U_{N}$ is want to control one of his appliances $L_{11}$. User send a command which is decoded by the SEN -system. SEN system already gets information about that load system which is storage in memory. Using user control system it commands to see that appliance working condition and reply to the user.

$$
U=G \times L^{H}
$$

The performance of measuring energy detector based on sensing probabilities. $P_{D}$ is the probability of considering frequency signal which is truly present. The desiring RF spectrum is large, so that we can formulate probability of spectrum as high as possible.

$$
\begin{gathered}
P_{D}=P_{r}\left(M>\lambda_{E D} \mid H_{1}\right) . \\
P_{D}=P_{r}\left(M=\mu \mid H_{1}\right) .
\end{gathered}
$$

Where $\left(H_{1}\right)$ is a hypothesis [9] such that,

$$
H_{1}: y(n)=s(n)+u(n)
$$

Where $s(n) \& u(n)$ refers to the signal to be detected and white Gaussian noise. At the time of receiving information in Smart energy meter, the error signal is considered as white noise. By solving equation (17) and (27) we get;

$$
u(n)=s(n)-e(n)
$$

Finally energy detection methods converges to [13] 


$$
P_{D}=1-\Gamma\left(L_{f} L_{t}, \frac{\mu}{\sigma^{2} s^{-\sigma_{e}^{2}}}\right)
$$

Where $\lambda_{E}$ is the decision threshold and $\Gamma(a, x)$ is the incomplete gamma function. SNR is defined as the ratio of the primary user's signal to noise power.

$$
S N R=\sigma^{2} s / \sigma_{e}^{2}
$$

The waveform based sensing metric can be obtained as,

$$
M=\operatorname{Re}\left[\sum_{n=1}^{N} u(n) s^{*}(n)\right] .
$$

Where the * represents the conjugate operation. Let LMS system works in unlicensed frequency with maximum probability. The final equation for primary user which can detect through LMS algorithm, which is given by;

$$
U=G^{H} \times L \times\left(1-\Gamma\left(L_{f} L_{t}, \frac{w(n+1)-\mathrm{w}(n)}{\mathrm{u}(n) e^{*}(n)\left(\sigma^{2}{ }_{s}-\sigma^{2} e^{2}\right)}\right)\right) \ldots
$$

\section{x. SIMULATION RESULTS}

The various temperature effects on fuel cell and li-ion battery systems are shown in [figure 8] and [figure 9] respectively. The graph was plotted by using some experimental results and MATLAB Simulink Software.

Increasing temperature of fuel cell stack results the better perform but due to rising temperature, more water will be evaporates during the chemical reactions. Whereby, the membrane dries up more and more. Which increases the ohmic resistance as a results ionic conductivity decreases. At too low temperatures, the catalytic process is restrained. To test the temperature effects, we increased the temperature from $40^{\circ} \mathrm{C}$ to $85^{\circ} \mathrm{C}$, then the output voltage of fuel cell increased gradually.

On the other hand, we increased the temperature from $-20^{\circ} \mathrm{C}$ to $30^{\circ} \mathrm{C}$, the output voltage of li-ion battery gradually decreased. Thus, higher temperature is not favorable for Lithium-ion battery.

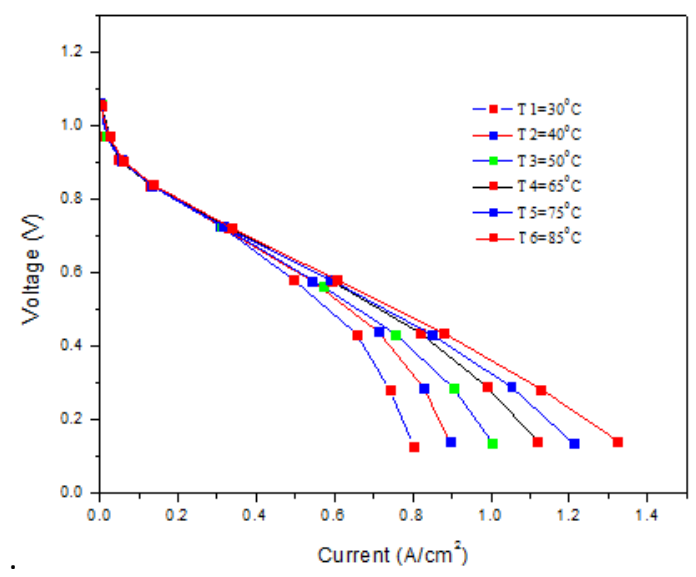

Figure 8: Temperature Effect on PEM Fuel Cell

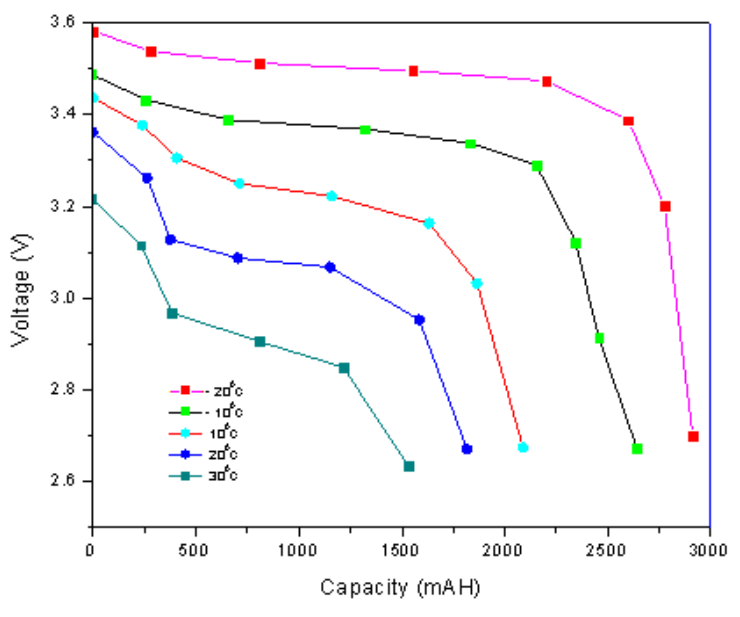

Figure 9: Temp Effect on Li- ion Battery

[Figure 10] shows the relationship between the probability of detection and probability of false alarm with different number of users. When number of users increases in the network, due to energy detection sensing technique the probability of signal detection gets enhanced. In this test, we increased the number of users $(\mathrm{N})$ from 20 to 90 . When the number of users increased from lower to higher level, probability of detection is also increased. So that, more number of user can be monitor and control one at a time.

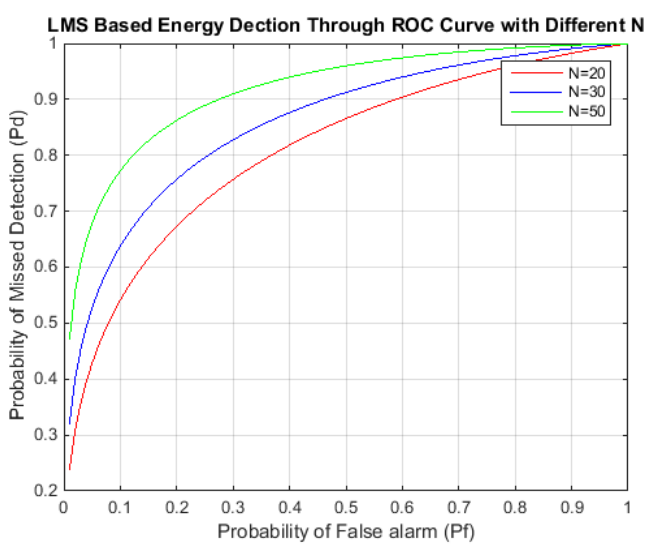

Figure 10: LMS Sensing signal for each end-user

\section{CONCLUSION}

From the above simulation results, we can conclude that, neither minimum nor maximum temperature is suitable for the performance of hybrid fuel cell and li-ion battery system. So for the better performance, we need to observed and control the effects of temperature through monitoring and control unit. In the case of fuel cell, by humidifying the supplied oxygen or cooling can prevent from overheating effects. Actually, temperature from $40^{\circ} \mathrm{C}$ to $85^{\circ} \mathrm{C}$, PEM fuel cell provides higher capacity. Similarly, higher temperature is not suitable for better li-ion performance. So, when the temperature is increased, we can control by proper cooling system.

Maximum numbers of radio frequencies are allocated through licensing. So, the existing spectrums become shortage due to increased number of wireless applications and the number of users. Thus, we can't properly monitor and control our system. To overcome from these situation LMS estimation is used in coded form and implemented 
through cognitive radio environment. There are maximum number of allocated frequency which are unused in each licensed frequency spectrum. LMS estimation has high probability of signal detection characteristics in cognitive radio environment, so we can have access to these unused frequency spectrum. That's why, maximum number of secondary user will be increased. As a result, frequency shortage problem as well as system hanging problem will be solved. Which also increased the efficiency of smart monitoring system.

\section{Acknowledgment}

This work was supported by KEPCO Research Institute grant funded by Korea Electric Power Corporation (R16DA11).

\section{References}

[1] J. Peng and S.J. Lee, 'Numerical Simulation of Proton Exchange Membrane Fuel Cells at High Operating Temperature', Journal of Power Sources, Vol. 162, N², pp. 1182 - 1191, 2006

[2] Chang, Hui Il, and Prakash Thapa. "A Study of Monitoring and Operation for PEM Water Electrolysis and PEM Fuel Cell Through the Convergence of IoT in Smart Energy Campus Microgrid." 한국융합학회논문지 7.6 (2016): 13-21

[3] Linares, N., Silvestre-Albero, A. M., Serrano, E., Silvestre-Albero, J., \&García-Martínez, J. (2014). Mesoporous materials for clean energy technologies. Chemical Society Reviews, 43(22), 7681-7717.

[4] Lin, Feng, Dennis Nordlund, Isaac M. Markus, Tsu-ChienWeng, Huolin L. Xin, and Marca M. Doeff. "Profiling the nanoscale gradient in stoichiometric layered cathode particles for lithium-ion batteries." Energy \& Environmental Science 7, no. 9 (2014): 3077 3085.

[5] Wei, Zhongbao, BinyuXiong, DongxuJi, and King Jet Tseng. "Online State of Charge and Capacity Dual Estimation with a Multi-timescale Estimator for Lithium-ion Battery." Energy Procedia 105 (2017): 2953-2958.

[6] Shuaib, Khaled, et al. "Cognitive Radio for Smart Grid with Security Considerations." Computers 5.2 (2016):

[7] Ghassemi A, Bavarian S, Lampe L. Cognitive radio for smart grid communications. In: Proc. of First IEEE International Conference on Smart Grid Communications, 2010:297-302.

[8] Dehalwar, Vasudev, Mohan Kolhe, and Sunita Kolhe. "Cognitive radio application for smart grid." Int. Journal of Smart Grid and Clean Energy 1.1 (2012):79-84.

[9] I. F. Akyildiz, W. Y. Lee, M. C. Vuran, and S. Mohanty, Next generation/dynamic spectrum access/cognitive radio wireless networks: A survey, Computer Networked, 50 (2006) 2127-2159.

[10] IEEE Standard Definitions and Concepts for Dynamic Spectrum Access: Terminology Relating to Emerging Wireless Networks, System Functionality, and Spectrum Management, IEEE Std. 1900.12008, Sep. 2008

[11] V.C. Gungor and D. Sahin, Cognitive radio networks for smart grid applications: A Promising Technology to Overcome Spectrum Inefficiency. June 2012 IEEE Vehicular Technology Magazine, 4146.

About Author (s):

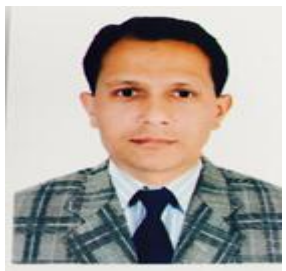

Currently studying $\mathrm{PhD}$ degree in Mokpo National University, South Korea. Interested in Li-ion Battery, Fuel Cell and Smart Grid Energy System etc.

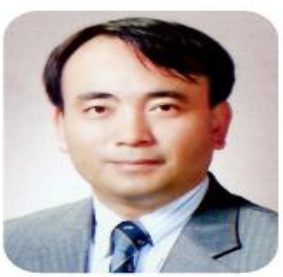

Working as a Professor in Mokpo National University, South Korea, since 1995

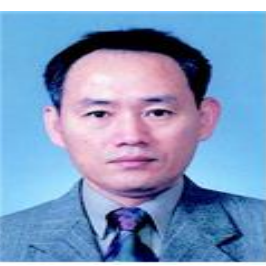

Working as a Professor in Mokpo National University, South korea, since 1990

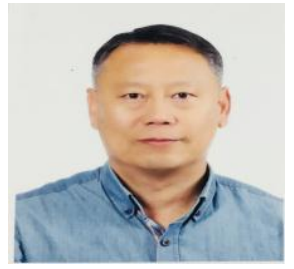

Currently working as a researcher in Mokpo National University, South Korea, since 2017

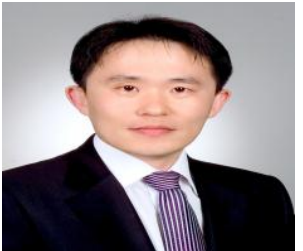

Working as a Assistant Professor in Mokpo Science University, South Korea, since 2010 